\title{
7. An Elementary Proof of the Grace's Theorem on Double Sixers.
}

\author{
By Asajiro IchroA.
}

(Recevied February 1, 1932)

Theorem: If we have five lines $a, b, c, d$, e intersecting a given ine $p$, then any four of them as $a, b, c, d$ have another common intersecting line; then we get such five lines and by the theorem of double six, we know that these five lines are met by another line $F$. If we take six lines $a, b, c, d, e, f$ meeting a given line $p$, then from each set of five we get a line like $F$ and these six lines are all met by one and the same line.

This theorem was proved first by J. H. Grace ( ${ }^{I}$ ) and discussed from various points of view by T. L. Wren $\left({ }^{2}\right), \mathrm{H} . \mathrm{F}$. Baker $\left({ }^{3}\right)$ and T. $\operatorname{Kubota}\left({ }^{4}\right)$. Here we will give an elementary proof in the same manner as A. C. Dixon proved the theorem of double six $\left({ }^{5}\right)$.

If we denote another line intersecting $c, d, e, f$ by $(a b)$ and so on, then we have a common intersecting line $A$ of $(a b),(a c),(a d),(a e),(a f)$; a common intersecting line $B$ of $(a b),(b c),(b d),(b e),(b f)$; a common intersecting line $C$ of $(a c),(b c),(c d),(c e),(c f)$; a common intersecting line $D$ of $(a d),(b d),(c d),(d e),(d f)$; a common intersecting line $E$ of $(a e),(b e),(c e),(d e),(e f)$; a common intersecting line $F$ of $(a f)$, (bf), $(e f),(d f),(e f)$. From the fact $\left({ }^{6}\right)$ that there is a common intersecting line of $A, B, C, D, e$ and $f$, we can prove the theorem by making use of the theorem of double six. Therefore it will suffice to prove the existence of the common intersecting line of $A, B, C, D, e$ and $f$ (Kubota's Lemma).

As a common intersecting line of the four lines $C, D, e, f$ is $(a b)$, we will denote another common intersecting line by $x$.

\footnotetext{
(2) T. L. Wren, Proc. London Math. Soc., 15 (1916).

$\left({ }^{3}\right)$ H. F. Baker. Proc London Math. Soc., 15 (1916).

( $\left.{ }^{4}\right)$ T. Kubota, Sciencé Reports, Tôhoku Imp. University, I, 6 (1917).

(5) A. C. Dixon, Quarterly Journal of Math., 40 (1909).

(s) T. Kubota, Lemma I, loe. cit.
} 16 (1898).

(1) J H. Grace, Circle, Spheres and Linear Complexes, Trans. Camb. Phil. Soc., 
Since the lines $x, D$ meet, the four points $x e, x f, D(c d), D(b d)$ lying on the four sides of the quadrilateral $e(c d), e(b d), f(b d), f(c d)$ (herein $x e$ denotes the meet of the two lines $x$ and $e$ ) are in a plane, and therefore

$$
\frac{[e(c d) \cdot x e]}{[x e \cdot e(b d)]} \frac{[e(b d) \cdot D(b d)]}{[D(b d) \cdot f(b d)]} \frac{[f(b d) \cdot x f]}{[x f \cdot f(c d)]} \frac{[f(c d) \cdot D(c d)]}{[D(c d) \cdot e(c d)]}=1
$$

where $[x e \cdot e(b d)]$ denotes the distance between the two points $x e$ and $e(b d)$.

Similarly by the consideration of the lines $x, c$ and the quadrilateral $e(c d), e(b c), f(b c), f(c d)$

$$
\frac{[e(b c) \cdot x e]}{[x e \cdot e(c d)]} \frac{[e(c d) \cdot C(c d)]}{[C(c d) \cdot f(c d)]} \frac{[f(c d) \cdot x f]}{[x f \cdot f(b c)]} \frac{[f(b c) \cdot C(b c)]}{[C(b c) \cdot e(b c)]}=1 .
$$

By the consideration of the lines $x, B$ and the quadrilateral $e(b d)$, $e(b c), f(b c), f(b d)$, and using the notation

$$
\frac{[e(b d) \cdot x e]}{[x e \cdot e(b c)]} \frac{[e(b c) \cdot B(b c)]}{[B(b c) \cdot f(b c)]} \frac{[f(b c) \cdot x f][f(b f \cdot f(b d)]}{[B(b d) \cdot e(b d)]}=X
$$

we have as the condition for the intersection of $x$ and $B$

$$
X=1 \text {. }
$$

Again by the consideration of $(a d), D$ and the quadrilateral $e(c d)$, $e(b d), f(b d), f(c d)$, we have

$$
\frac{[f(b d) \cdot D(b d)][e(b d) \cdot e(a d)]}{[D(b d) \cdot e(b d)][e(a d) \cdot e(c d)]} \frac{[e(c d) \cdot D(c d)]}{[D(c d) \cdot f(c d)]} \frac{[f(c d) \cdot f(a d)]}{[f(a d) \cdot f(b d)]}=1
$$

and by the consideration of $(a c), C$ and the quadrilateral $e(c d), e(b c)$, $f(b c), f(c d)$

$$
\frac{[f(c d) \cdot C(c d)]}{[C(c d) \cdot e(c d)]} \frac{[e(c d) \cdot e(a c)]}{[e(a c) \cdot e(b c)]} \frac{[e(b c) \cdot C(b c)]}{[C(b c) \cdot f(b c)]} \cdot \frac{[f(b c) \cdot f(\alpha c)]}{[f(a c) \cdot f(c d)]}=1,
$$

and by the consideration of $(a b) B$ and the quadrilateral $e(b d), e(b c)$, $f(b c), f(b d)$

$$
\frac{[f(b c) \cdot B(b c)\rceil[e(b c) \cdot e(a b)]]}{[B(b c) \cdot e(b c)]} \frac{[e(b d) \cdot B(b d)]}{[e(a b) \cdot e(b d)][B(b d) \cdot f(b d)][f(a b) \cdot f(b c)]}=1 .
$$

And by the consideration of $d,(b c)$ and the quadrilateral $e(a c), e(a b)$, $f(a b), f(a c) ; c,(b d)$ and the quadrilateral $e(a d), e(a b), f(a b), f(a d) ; b,(c d)$ and the quadrilateral $e(a d), e(a c), f(a c) f(a d) ; p, d$ and the quadrilateral $e(a c), e(a b), f(a b), f(a c) ; p, c$ and the quadrilateral $e(a d), e(a b), f(a b), f(a d)$; $p, b$ and the quadrilateral $e(a d), e(a c), f(a c), f(a d)$ we have

$$
\frac{[e(a c) \cdot e(b c)]}{[e(b c) \cdot e(a b)]} \frac{[e(a b) \cdot d(a b)]}{[d(a b) \cdot f(a b)]} \frac{[f(a b) \cdot f(b c)]}{[f(b c) \cdot f(a c)]} \frac{[f(a c) \cdot d(a c)]}{[d(a c) \cdot e(a c)]}=1
$$




$$
\begin{aligned}
& \frac{[e(a b) \cdot e(b d)]}{[e(b d) \cdot e(a d)]} \frac{[e(a d) \cdot c(a d)]}{[c(a d) \cdot f(a d)]} \frac{[f(a d) \cdot f(b d)]}{[f(b d) \cdot f(a b)]} \frac{[f(a b) \cdot c(a b)]}{[c(a b) \cdot e(a b)]}=1, \\
& \frac{[e(a d) \cdot e(c d)]}{[e(c d) \cdot e(a c)]} \frac{[e(a c) \cdot b(a c)]}{[b(a c) \cdot f(a c)]} \frac{[f(a c) \cdot f(c d)]}{[f(c d) \cdot f(a d)]} \frac{[f(a d) \cdot b(a d)]}{[b(a d) \cdot e(a d)]}=1, \\
& \frac{[f(a b) \cdot d(a b)]}{[d(a b) \cdot e(a b)]} \frac{[e(a b) \cdot p e]}{[p e \cdot e(a c)]} \frac{[e(a c) \cdot d(a c)]}{[d(a c) \cdot f(a c)]} \frac{[f(a c) \cdot p f]}{[p f \cdot f(a b)]}=1, \\
& \frac{[f(a d) \cdot c(a d)]}{[c(a d) \cdot e(a d)]} \frac{[e(a d) \cdot p e]}{[p e \cdot e(a b)]} \frac{[e(a b) \cdot c(a b)]}{[c(a b) \cdot f(a b)]} \frac{[f(a b) \cdot p f]}{[p f \cdot f(a d)]}=1, \\
& \frac{[f(a c) \cdot b(a c)]}{[b(a c) \cdot e(a c)]} \frac{[e(a c) \cdot p e]}{[p e \cdot e(a d)]} \frac{[e(a d) \cdot b(a d)][b(a d) \cdot f(a d)]}{[f(a d) \cdot p f]}=1 .
\end{aligned}
$$

Multiplying these twelve equations side by side we have

$$
1=X
$$

which: expresses the fact that $B$ meets $x$. And it can similarly be proved that $A$ also meets w. Q.E.D.

Oct. $21^{\text {st }}, 1931$. 